\title{
Simulate the single-throw electric upcut shears operation in Mathcad
}

\author{
Anatoly Drozdov* \\ Moscow State National Research University of Civil Engineering, 129337, Moscow, Russia
}

\begin{abstract}
The relevance of sheet processing technology, which finds increasing application, is given. Efficient equipment for sheet metal processing and sheet mounting is justified and proposed. This article deals with upcut shears' operation. The solution to the mathematic model of manual upcut shears using Mathcad is provided.
\end{abstract}

\section{Introduction}

The technology development for the use of light steel structures modules with a long service life in the construction, low specific gravity, low operating cost and others, in addition to their improvement, sets the task of rationally choosing the mechanization means by which these works are carried out.

In addition to lifting equipment, a mechanized tool (hand-held machines) is important here. First of all, we are talking about the shears and threading machines, which are used when fitting in place and installing products.

The technological processes implemented by these machines are characterized by the vibration and vibration shock modes. Reducing the vibration level transmitted to the operator makes it possible to extend its operation time with the machine. In this regard, the entire technology effectiveness is increased. This requires developers of manual machines to use mathematical models at the stage of their creation. With regard to thread-wrapping machines, these issues are covered, in particular, in the works: $[1,2,3]$. This article deals with the approach to manual upcut shears, the production of which is carried out by a number of leading companies $[4,5]$.

Certain aspects of this task are reflected, in particular, in the works: $[6,7,8,9]$.

Upcut shears are designed for separation of sheet metal with ultimate strength up to 40 $\mathrm{MPa}$, thickness up to $6.0 \mathrm{~mm}$. Their movable working member reciprocates at a frequency of $18 \ldots 22 \mathrm{~Hz}$ and the oscillation range are slightly less than the maximum thickness of the sheet to be separated (Fig. 1).

According to the type of energy used, the shears are electric and pneumatic in the drive, and according to the type of working elements that determine the type of metal treatment (upcut, pressure) - knife (slotted are considered as a special case of knife) and upcut.

A type of upcut shears are the edge formers - machines that provide preparation of the parts edges for welding, with a thickness of the treated sheet up to $30 \mathrm{~mm}$.

* Corresponding author: drozdovan@mgsu.ru 
Portable shears have a higher upcut speed, do not give material to chip, but they provide a length of strips only from the edges, bending them somewhat.

Nibble shears are made by upcut the sheet in an arbitrary place with a minimum upcut radius, which ensures their use for upcut holes of various shapes in smooth and corrugated sheets.

\section{Research methodology}

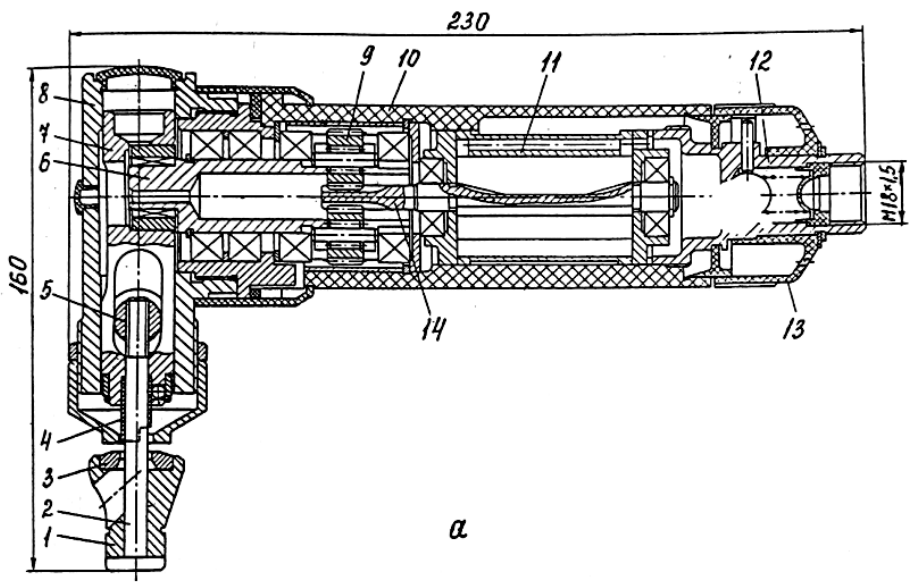

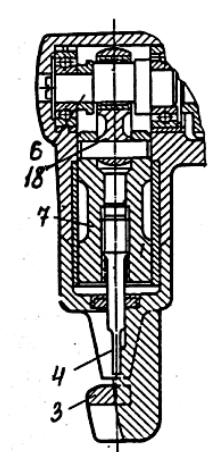

b

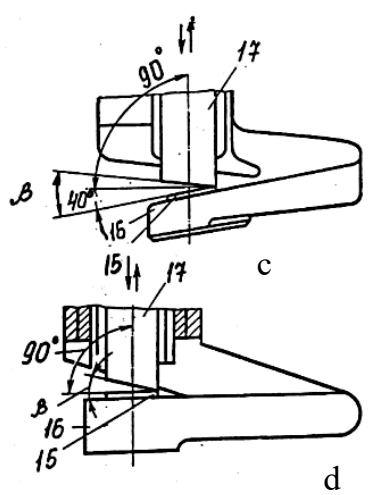

d

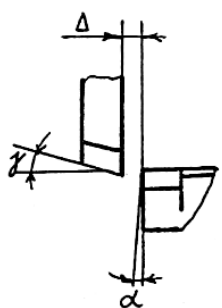

Fig. 1. Manual pneumatic shears: a, $b$ - upcut; $c, d$ - knife: 1 - a matrix holder; 2 - rod; 3 - a matrix; 4 - punch; 5 - cross-arm; 6 - in length; 7 - slider; 8 - head housing; 9 - planetary reduction gear box; 10 - haft case; 11 - pneumatic motor; 12 - launcher; 13 - trigger handle; 14 - rotor shaft; 15 fixed knife; 16 - holder (snail); 17 shows a slider with a movable knife; 18 - rocking collar; $\beta$ - sizing angle; $\gamma$ - rear angle; $\Delta$ - clearance between knives; $\alpha$ - front angle

Dynamic models of PM certain types of drives (mosaic grinding, upcut shears, etc.) with sufficient accuracy can be represented by the circuits with rigid kinematic connections.

In this case, the equivalent mechanical drive system can be represented as a single-mass system with a mass driven, for example, to the motor shaft. In this case, the rotation angle of the motor $\alpha$ will be the generalized coordinate of the system. Total moment of engine inertia and reduced mass $I=I_{m o t}+I_{p r}$. In the transient mode of the drive operation, the equation of its motion will take the form: 


$$
M_{d}(\omega)=M_{c}(\omega)+M_{d y n}
$$

where $M_{d}(\omega)$ - is the moment of the engine determined by its mathematical model or by the available mechanical characteristic;

$M_{c}(\omega)$ - is the moment of resistance forces determined by the type of work operation performed and characteristics of the processing object;

$\omega=d \alpha / d t=\dot{\alpha}-$ is a shaft rotation speed;

$M_{d y n}$ - defines the dynamic moment ensuring change of engine rotation speed.

Depending on moment of inertia I ( - const $I=I(\alpha)$ ) value $M_{\text {dyn }}$ will be defined differently.

If $I$ - const

$$
M_{d y n}=I(d \omega / d t)
$$

If $I=I(\alpha)$, it is characteristic, in particular, for the drives with crank-rod and eccentric converter mechanisms, value $M_{d y n}$ we define from the Lagrange equation of the second kind in the form:

$$
M_{d y n}=\frac{d}{d t} \cdot \frac{d A}{d \omega}-\frac{d A}{d \alpha}
$$
drive.

where $A=I(\alpha)\left(\omega^{2} / 2\right)$ - is the kinetic energy stored by the mechanical part of the

Having differentiated, we get:

$$
M_{d y n}=I(\alpha) \frac{d \omega}{d t}+\frac{\omega^{2}}{2} \times \frac{d I(\alpha)}{d \alpha}
$$

The equations (1) and (2) or (1) and (4), along with the specified initial conditions, give a complete description of the drive with rigid kinematic constraints. In steady state operation $\left(\frac{d \omega}{d t}=0\right)$ the equation of the drive motion will be:

$$
M_{d}(\omega)=M_{c}(\omega)
$$

Based on the given dependences, we obtain the equation of motion of the drive of vibration-type hand shears with an eccentric conversion mechanism ( $\mathrm{r}$ is the eccentricity value) and analyse it. Let us choose the value of the eccentric shaft rotation angle - $\alpha$ as the generalized coordinate. We will take the extreme right position of the eccentric shaft as the starting point. From here we will count the positive $\mathrm{x}$ coordinate of the slider translational movement. In this case, the connection between them is as follows:

$$
x=r(1-\cos \alpha)
$$

The value of the machine drive parts' inertia, reduced to the eccentric shaft axis:

$$
I_{p r}=I_{p} i^{2}+m r^{2} \sin ^{2} \alpha
$$

Where $I_{p}$ - is the eccentric shaft drive inertia moment reduced to the axis of the armature-rotor;

$m-$ is the mass of the translationally moving parts of the drive.

The moment of resistance forces, reduced to the axis of the eccentric shaft,

$$
M_{c}=\operatorname{Pr}_{1} \sin \alpha
$$

Where $P$ - is the force generate don the tool when performing the working process of upcut or punching;

$\eta_{1}-$ is an efficiency of the conversion mechanism. 
Depending on the type of excitation winding (serial, parallel) of collector single-phase electric motors, their external mechanical static characteristic can be written by the corresponding expressions

$$
M_{m o t}=\left\{\begin{array}{c}
a_{0} / \dot{\alpha}_{m o t}-a_{1} \\
b_{0}-b_{1} \dot{\alpha}_{e n g}
\end{array}\right.
$$

Where $a_{0}, a_{1}, b_{0}, b_{1}$ - are the constant coefficients.

Let us take $\mathrm{M}_{\text {mot }}\left(\dot{\alpha}_{m o t}\right)$ in the form (9b), where $b_{0}=M_{0}-$ is the moment of the "inhibited" engine - the conditional point of intersection of the linearized characteristic with the axis of moments; $b_{1}$ - the tangent of the slope of the characteristic to $\dot{\alpha}_{\text {mot }}$.

In this case, from (1), taking into account (4), where $I=I_{s l}$, [determined from (7)], as well as (8), we obtain the equation of the drive motion in the form

$$
\ddot{\alpha}\left(m r^{2} \sin ^{2} \alpha+I_{p} i^{2}\right)+0,5 \dot{\alpha}^{2} m r^{2} \sin 2 \alpha+b \eta_{1} \dot{\alpha}=M
$$

where $M=M_{0} i \eta_{2}-\operatorname{Pr} \eta_{1} \sin \alpha$;

$i, \eta_{2}$ - are the gear ratio of the gearbox and the eccentric shaft drive efficiency respectively.

For the convenience of performing dynamic analysis, we rewrite the obtained equation of the drive motion relative to the generalized coordinate $\mathrm{x}$, assuming $\dot{\alpha}=\omega$ - constand taking $P=c x+b \dot{x}$ ( $c-$ is the reduced stiffness of the stationary working body holder and a sheet of metal; $b$ - is the coefficient resistance), in the form:

$$
m_{s l} \ddot{x}+b \dot{x}+c x=F_{0}[1-\cos (\omega t+\varphi)]
$$

where $m_{s l} \quad-$ is the drive mass reduced to the slider; $\varphi-$ is the phase shift angle between the displacement and the driving force; $\mathrm{F}_{0}-$ is the force amplitude in the slide

$$
h=b /\left(2 m_{s l},\right) ; 0 ;<\omega_{0} / \sqrt{2} ;>\omega_{0} / \sqrt{2} .
$$

\section{Discussion}

To create a mathematical model of Portable shears driven by an AC collector motor, we set the function of the moment of inertia brought to the shaft for the motion system $J(\varphi)$ according to (7), as well as the function of the resistance moment $M_{\mathcal{c}}(\varphi)$ according to (8):

$$
\begin{gathered}
\frac{d i_{a}}{d t}=\frac{U_{m}}{L_{a}} \sin \omega_{1} t-\frac{i_{a}}{L_{a}}\left(\sum R+\frac{k_{T} k_{\sigma} X_{a d} \sin \gamma}{k_{\mu}}\right)-\frac{C_{e}}{L_{a}}\left(\varphi_{\delta 1}+\varphi_{\delta 2} \operatorname{tg} \gamma\right) \omega \cos \alpha_{s h} \\
\frac{d i_{a}^{\prime}}{d t}=\frac{U_{m}}{L_{a}} \cos \omega_{1} t-\frac{i_{a}^{\prime}}{L_{a}}\left(\sum R+\frac{k_{T} k_{\sigma} X_{a d} \sin \gamma}{k_{\mu}}\right)-\frac{C_{e}}{L_{a}}\left(\varphi_{\delta 1}+\varphi_{\delta 2} \operatorname{tg} \gamma\right) \omega \cos \alpha_{s h} \\
\frac{d \omega}{d t}=\frac{p N}{2 \pi a J_{s l}(\varphi)} i_{a} \varphi_{\delta}-\frac{M_{0 n}}{J_{s l}(\varphi)}-\frac{k_{l}}{J_{s l}(\varphi)} \omega-\frac{k_{s q}}{J_{s l}(\varphi)} \omega^{2}-\frac{\psi(\varphi) M_{c}(\varphi)}{J_{s l}(\varphi)}
\end{gathered}
$$

The reduced moment of inertia

$$
J_{s l}(\varphi)=J+\left(J_{\text {red }}+m r^{2} \sin ^{2}(\varphi)\right) i_{\text {red }}^{2}
$$

$J-$ is the moment of the rotor inertia for the engine itself, $\mathrm{kg} \mathrm{m}^{2} ; J_{\text {red }}-$ shaft, $\mathrm{kg} \mathrm{m}^{2} ; m-$ - the angular coordinate of the rotor of the engine, I am glad; $i_{\text {red }}$ - gear ratio of reduction gear box.

The given moment of resistance (the blade coordinates are counted from the middle position, the upper dead point is located on the positive position, and the lower - on the negative axis) can be set as 


$$
M_{c}(\varphi)=\left\{\begin{array}{c}
\frac{P_{c u t}}{i_{\text {red }}}(r \eta|\sin (\varphi)|), \text { if } r \sin (\varphi)<h-r \\
\frac{C_{c u t}}{i_{\text {red }}}\left[\left(h+\frac{P_{c u t}}{C_{c u t}}-r\right)-r \sin (\varphi)\right](r \eta|\sin (\varphi)|) \text { if }\left\{\begin{array}{c}
r \sin (\varphi) \leq h+\frac{P_{c u t}}{C_{c u t}}-r \\
r \sin (\varphi) \geq h-r
\end{array}\right. \\
0, \text { in other cases }
\end{array}\right.
$$

$C_{c u t}-$ is the stiffness of fixed blade holder, N/m; $P_{c u t}-$ is upcut power, $\mathrm{N} ; \eta-$ is an actuator efficiency; $\mathrm{r}-$ is an eccentricity value, $\mathrm{m} ; \mathrm{h}-$ is the thickness of metal to be cut, $\mathrm{m}$. At the same time: relation $\mathrm{P}_{\text {cut }} / \mathrm{C}_{\text {cut }}$ - is the maximum holder deformation, $\mathrm{m}$; $\left(\mathrm{h}+\mathrm{P}_{\text {cut }} / \mathrm{C}_{\text {cut }}\right.$ - r) - is the blade coordinate at which the blade contacts the metal to be treated (the deformation process of the holder begins); $\mathrm{m}$; $(\mathrm{h}-\mathrm{r})$ - is the blade coordinate, at which the holder deformation is maximum (the metal separation process begins), $\mathrm{m}$.

Auxiliary operator:

$$
\psi(\dot{\varphi})=\left\{\begin{array}{l}
1, \text { if } \dot{\varphi}>0 \\
0, \text { if } \dot{\varphi} \leq 0
\end{array}\right.
$$

Since the blade coordinate is proportional to $\sin (\mathrm{a})$, the following replacement is true:

$$
\psi(-\cos (\varphi))=\left\{\begin{array}{l}
1, \text { if }[-\cos (\varphi)]>0 \\
0, \text { if }[-\cos (\varphi)] \leq 0
\end{array}(16)\right.
$$

\section{Summary}

The following is a simulation of the single-throw shears operation in the MathCAD

\begin{tabular}{|c|c|c|c|c|c|c|c|}
\hline \multirow{3}{*}{$\mathrm{J}:=0.609 \cdot 10^{-4}$} & $\mathrm{~N}: 1292$ & $\alpha \delta:=0.69$ & wf:=194 & $\delta:=0.5 \cdot 10^{-3}$ & $\mathrm{k} \delta:=1.178$ & $\mathrm{a}:=1$ & \multirow{2}{*}{$\mathrm{UH}:=220$} \\
\hline & $\mathrm{B} \delta \mathrm{cr}:=0.375$ & Iacr:=1.72 & $\Phi \delta \mathrm{sc}:=6.25 \cdot 10^{-4}$ & $\alpha 1:=8.32 \cdot 10^{-4}$ & $\beta 1:=0.0065$ & $\alpha 2:=0.371$ & \\
\hline & & & & & & & $\mathrm{p}:=2$ \\
\hline$\beta 2:=0.008$ & $\mathrm{X} \sigma \mathrm{a}:=1.727$ & $X \sigma f:=1.56$ & $\mathrm{Xaq}:=11.5$ & Xad: $=130$ & $\Delta \mathrm{Um}:=2.4$ & $\mathrm{Ra}:=2.21$ & $\mathrm{nh}:=8000$ \\
\hline $\mathrm{Rf}:=16.35$ & $\alpha h:=0$ & Ian:=3.15 & Ptrp: $=26.8$ & Pfr: $=0.86$ & Pcz2:=15.9 & Pza2:=3.8 & \\
\hline Ptrs $:=3$ & $\mathrm{P}_{2 \mathrm{H}}:=400$ & $\gamma:=0.15$ & $\mathrm{k} \sigma:=1.1$ & $\mathrm{Kt}:=0.151$ & $\mathrm{~b} 0:=0.005$ & $\omega 1:=2 \pi \cdot 50$ & $1 \delta:=0.063$ \\
\hline
\end{tabular}
system.

\section{Electric motor parameters}

\section{Shears parameters}

$\begin{array}{lll}\mathrm{m}:=0.1 & - & \text { mass of linearly moving drive parts, } \mathrm{kg} \\ \mathrm{Jr}:=0.2 \cdot 10^{-4} & - & \text { moment of eccentric shaft inertia } \mathrm{kg}, \mathrm{m}^{2} \\ \mathrm{r}:=0.003 & - & \text { eccentricity } \mathrm{m} \\ \mathrm{ir}:=3 & - & \text { gear ratio } \\ \eta:=0.8 & - & \text { conversion mechanism efficiency ratio } \\ \mathrm{Pcut}:=1800 & - & \text { upcut force } \mathrm{H} \\ \mathrm{Ccut}:=900000 & - & \text { rigidity of the fixed blade holder and the metal being } \\ & & \text { processed } \mathrm{N} / \mathrm{M} \\ \mathrm{h}:=0.001 & - & \text { metal thickness, } \mathrm{m}\end{array}$




\section{Calculated coefficients and functions}

$$
\begin{aligned}
& \mathrm{Um}:=\sqrt{2} \cdot \mathrm{UH}_{\mathrm{H}}
\end{aligned}
$$

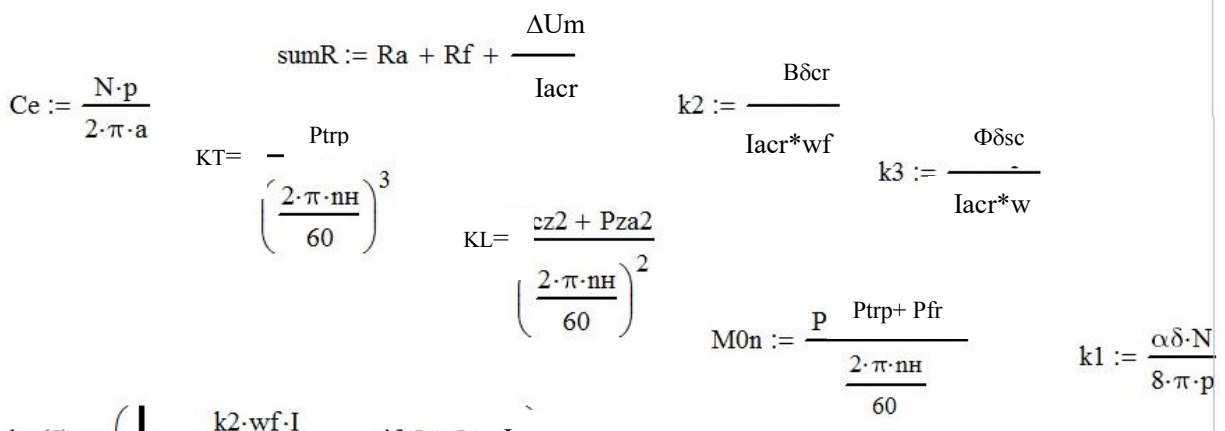

$$
\begin{aligned}
& \mathrm{k} \mu(\mathrm{I}):=\left(\begin{array}{l}
\frac{\mathrm{k} 2 \cdot \mathrm{wf} \cdot \mathrm{I}}{\alpha 1 \cdot \operatorname{atan}(\beta 1 \cdot \mathrm{I} \cdot \mathrm{wf})} \\
1 \text { otherwise }
\end{array}\right) \\
& \mathrm{La}(\mathrm{I}):=\left[\mathrm{X} \sigma \mathrm{a}+\mathrm{X} \sigma \mathrm{f}+\frac{\mathrm{Xaq}+\mathrm{Xad} \cdot(\mathrm{k} \sigma \cdot \mathrm{kt} \cdot \cos (\gamma) \cdot \sin (\alpha \mathrm{\Psi}))}{\mathrm{k} \mu(\mathrm{I})}\right] \cdot \frac{1}{\omega 1} \\
& \varphi \delta(\mathrm{I}):=\mid \begin{array}{l}
\mathrm{k} 3 \cdot \mathrm{wf} \cdot \mathrm{b} 0 \cdot 1 \delta \cdot \mathrm{I}-\mathrm{b} 0 \cdot 1 \delta \cdot \frac{\mathrm{k} 3 \cdot \mathrm{I} \cdot(\mathrm{k} 1+\mathrm{wf})-\alpha 2 \cdot \mathrm{atan}[\beta 2 \cdot \mathrm{I} \cdot(\mathrm{wf}+\mathrm{k} 1)]}{6} \text { if } \text { Iacr } \\
\mathrm{b} 0 \cdot 1 \delta \cdot \operatorname{atan}(\beta 2 \cdot \mathrm{I} \cdot \mathrm{wf})-\mathrm{b} 0 \cdot 1 \delta \cdot \frac{2 \cdot \alpha 2 \cdot \operatorname{atan}(\beta 2 \cdot \mathrm{I} \cdot \mathrm{wf})-\mathrm{k} 3 \cdot \mathrm{I} \cdot(\mathrm{wf}-\mathrm{k} 1)-\alpha 2 \cdot \operatorname{atan}[\beta 2 \cdot \mathrm{I} \cdot(\mathrm{wf}+\mathrm{k} 1)]}{6} \text { otherwise }
\end{array}
\end{aligned}
$$

$$
\operatorname{Jpr}(\varphi):=\mathrm{J}+\left(\mathrm{Jr}+\mathrm{m} \cdot \mathrm{r}^{2} \cdot \sin (\varphi)^{2}\right) \cdot \mathrm{ir}^{2} \quad \psi(\mathrm{V}):=\mid \begin{array}{ll}
1 & \text { if } \mathrm{V}>0 \\
0 & \text { otherwise }
\end{array}
$$$$
\operatorname{Mconp}(\varphi):=\mid \begin{aligned}
& \frac{\text { Prez }}{\text { ir }} \cdot(\mathrm{r} \cdot \eta \cdot|\sin (\varphi)|) \text { if } \mathrm{r} \cdot \sin (\varphi)<\mathrm{h}-\mathrm{r} \\
& \frac{\mathrm{Crez}}{\mathrm{ir}} \cdot\left[\left(\mathrm{h}+\frac{\operatorname{Prez}}{\mathrm{Crez}}-\mathrm{r}\right)+-\mathrm{r} \cdot \sin (\varphi)\right] \cdot(\mathrm{r} \cdot \eta \cdot|\sin (\varphi)|) \text { if } \mathrm{r} \cdot \sin (\varphi) \leq \mathrm{h}+\frac{\operatorname{Prez}}{\mathrm{Crez}}-\mathrm{r} \wedge \mathrm{r} \cdot \sin (\varphi) \geq \mathrm{h}-\mathrm{r} \\
& 0 \begin{array}{l}
\text { otherwise }
\end{array}
\end{aligned}
$$

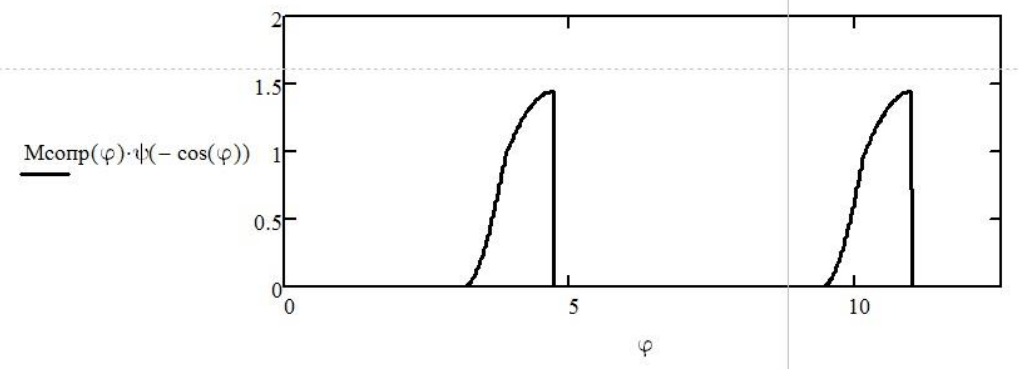

\section{References}

1. A.N. Drozdov, Improvement of assembly technique efficiency for installation of buildings and related structures in light building constructions. International Scientific Conference "Integration, Partnership and Innovation in Construction Science and Education" (IPICSE-2018) MATEC Web of Conferences. ISBN 978-5-7264-1896-4

2. A.N. Drozdov, Theoretical and empirical methods of estimating energy and force 
characteristics of impact wrenches, XXII International Scientific Conference on «Construction the Formation of Living Environment 2019» (FORM-2019)», Journal of Civil Engineering and Architecture, USA DOI: 10/17265/1934-7359

3. A.N. Drozdov, IOP Conf. Series: Materials Science and Engineering 869072022 (2020). doi:10.1088/1757-899X/869/7/072022

4. Information on https://www.trumpf.com/ru_RU/

5. Information on https://www.bosch.ru/products-and-services/industry-and-trades/

6. R.S. Zelenntsov, M.Sh. Weitman, Manual electrical machines for processing construction materials (Moscow, 1989).

7. Yu.V. Ratnikov, Study of workflows and dynamics of manual electronic shears, Lifting and transportation construction, road, track machines and robotic complexes materials of the 17th Moscow International Inter-University NTK, 2013

8. S.P. Rogovchenko, V.E. Strakovich, Computer methods for designing a mechanized instrument (MADI, 2018).

9. V.N. Markevich, Development of a methodology for assessing the level of safety of elevators, Collection of reports of the XXIV Moscow International Inter-University Scientific and Technical Conference of students, undergraduates, graduate students and young scientists, 2020.

10. A.F. Tikhonov, V.N. Batuev, A.N. Drozdov, Electric drive of a construction mechanized tool. Tutorial (MGSU-LLC AiPiErMedia, 2017).

11. A.N. Drozdov, Advances in Intelligent Systems and Computing, Springer Publishing House 692, 750-759 (2019). DOI: https://doi.org/10/007/978-3-319-70987-1-79.

12. A.N. Drozdov, V.V. Stepanov, Engineering of Impulse Mechanism for Mechanical Hander Power Tools, The 4 th Internation Conference on Advanced Engineering and Techology (4th ICAET) December 15-17 (2017) Incheon Korea. DOI: 10.1088/1757/$899 \mathrm{x} / 317 / 1 / 012034$

13. A.N. Drozdov, V.V. Stepanov, Development of mathematical models of hand machines for assembly work. Interstroymech-2018: collection of reports of the XXI International Scientific and Technical Conference (Moscow, October 8-12, 2018) ISBN 978-5-72641896-4 (2018). https://doi.org/10.1051/e3sconf/201997 05028

14. R.R. Sharapov, Bulletin of BSTU named after V.G.Shukhov 1, 65-69 (2011). 